\title{
Quantum computation by measurements
}

\author{
Debbie W. Leung \\ Institute of Quantum Information, MSC 10\%-81, Caltech, Pasadena, CA 91125, USA \\ wcleung@caltech.edu
}

(October 31, 2003)

\begin{abstract}
We first consider various methods for the indirect implementation of unitary gates. We apply these methods to rederive the universality of 4-qubit measurements based on a scheme much simpler than Nielsen's original construction quant-ph/0108020. Then, we prove the universality of simple discrete sets of 2-qubit measurements, again using a scheme simplifying the initial construction quant-ph/0111122. Finally, we show how to use a single 4-qubit measurement to achieve universal quantum computation, and outline a proof for the universality of almost all maximally entangling 4-qubit measurements.
\end{abstract}

\section{Introduction}

Studying the resources required for universal quantum computation is important not only for its realization but also for our theoretical understanding of what makes quantum computation so powerful. The standard model of quantum computation[1] requires a well defined and isolated Hilbert space. Universal computation further requires the ability (1) to prepare a fiducial initial state, (2) to implement a universal set of gates in a quantum circuit, and (3) to perform strong measurements. A set of quantum gates is universal if any unitary evolution can be approximated to arbitrary accuracy by a circuit involving those gates only. 2] When it is possible to perform a universal set of gates, it suffices to prepare the $|0\rangle$ state and to measure along the computation basis $\{|0\rangle,|1\rangle\}$ in conditions (1) and (3).

We describe some universal sets of gates that are relevant to the current discussion. The first one is the set of all 2-qubit gates. 3. The second set consists of all 1-qubit gates and only one 2-qubit gate, the controlled-NOT (СNOT).4 These two continuous sets of gates generate any unitary operation exactly. There are also finite, discrete universal sets that generate any operation to arbitrary accuracy. For example, the CNOT, the phase gate, the Hadamard gate, and the $\frac{\pi}{8}$ gate form a universal set. [5] Also, almost any single 2-qubit gate is universal. 6, 6, 8,

Other computation models have been built upon the standard model, so as to achieve fault tolerance or to adapt to promising physical systems. In these models, only some unitary gates can be easily performed and they do not form a universal set. In the context of fault tolerant quantum computation, Shor pioneered a recipe that indirectly performs the Toffoli gate using an ancilla, measurements, and some other gates. [9] The method was generalized [10, 11] by understanding the connection to teleportation. 12] The generalized method was applied to quantum computation schemes based on linear optics [13 and exchange interactions [14.

More recently, two different models of quantum computation based only on measurements are proposed. Raussendorf and Briegel[15] have proposed a "1-way quantum computer" which starts with a cluster state [16] of certain size and uses only 1-qubit measurements. The cluster state can be replaced by a circuit-dependent initial state that takes 4-qubit measurements to prepare. Independently, Nielsen [17 extended the indirect methods of performing unitary gates to obtain a quantum computation model using only measurements on up to 4 qubits. 
The central result in Ref. [17] is a method to perform the universal set of all two-qubit gates using 4-qubit measurements only. Focusing on the smaller set of 1-qubit gates and the CNOT, Fenner and Zhang 18 and independently Leung and Nielsen[19] showed that 3-qubit measurements are universal. Subsequently, Leung [20] showed that 2-qubit measurements are sufficient. This is the minimal number of qubits to be measured jointly in order to achieve universality because measurements are the only means of interaction. This result parallels the universality of 2 -qubit gates 3 in the standard model.

In this paper, we simplify and extend the results in Refs. [17] and [20. We systematically consider indirect implementation of unitary gates, including the method proposed in Ref. 10] and a new method that partially collapses the hierarchy of unitary gates proposed in Ref. [10]. We proceed to first rederive the universality of 4-qubit measurements [17] based on a much simpler scheme. Second, we rederive the result in Ref. [20] that 2-qubit measurements are universal. The current construction differs from Ref. [20] in using deterministic resources, similar to the 1-way quantum computer [15. Third, we prove that a single 4-qubit measurement can be universal, and we prove an analogue of the result that almost any 2-qubit gate is universal. [8, 6, 17]

\section{Indirect Implementations of Quantum Gates}

We first review the Pauli and Clifford groups (see Refs. 21, 22, 23] for example). Let $\sigma_{1,2,3}$ or $X, Y, Z$ be the Pauli operators and $\sigma_{0}$ be the $2 \times 2$ identity matrix,

$$
\sigma_{0}=\left(\begin{array}{ll}
1 & 0 \\
0 & 1
\end{array}\right), \sigma_{1}=\left(\begin{array}{ll}
0 & 1 \\
1 & 0
\end{array}\right), \sigma_{2}=\left(\begin{array}{cc}
0 & -i \\
i & 0
\end{array}\right), \sigma_{3}=\left(\begin{array}{cc}
1 & 0 \\
0 & -1
\end{array}\right) .
$$

The Pauli group is generated by $\sigma_{j}$ acting on each qubit. The Clifford group consists of unitary operators that conjugate Pauli operators to Pauli operators. The Clifford group is generated by the CNOT, the phase gate $\mathrm{P}=e^{-i \frac{\pi}{4} Z}$, and the Hadamard gate $\mathrm{H}=\frac{1}{\sqrt{2}}(X+Z)$.

A crucial element in performing unitary gates indirectly is teleportation [12 that transmits a qubit $|\psi\rangle=$ $a|0\rangle+b|1\rangle$ using the following circuit:

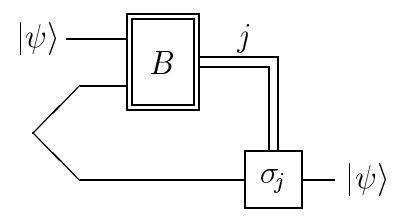

In the circuits throughout this paper, time goes from left to right, single lines denote qubits, double lines denote classical data, single- and double-lined boxes respectively denote unitary gates and measurements. A gate that is connected to a measurement box by a double line is performed conditioned on the measurement outcome. Two qubits connected in the left are initially in a maximally entangled state $\left|\Phi_{0}\right\rangle=\frac{1}{\sqrt{2}}(|00\rangle+|11\rangle)$. The Bell measurement, labeled $B$, is along the Bell basis:

$$
\begin{aligned}
& \left|\Phi_{0}\right\rangle=\frac{1}{\sqrt{2}}(|00\rangle+|11\rangle), \quad\left|\Phi_{3}\right\rangle=\frac{1}{\sqrt{2}}(|00\rangle-|11\rangle), \\
& \left|\Phi_{1}\right\rangle=\frac{1}{\sqrt{2}}(|01\rangle+|10\rangle), \quad\left|\Phi_{2}\right\rangle=\frac{1}{\sqrt{2}}(|01\rangle-|10\rangle) .
\end{aligned}
$$

Equation (21) can be verified by rewriting the initial state $|\psi\rangle\left|\Phi_{0}\right\rangle$ as $\frac{1}{2} \sum_{j}\left|\Phi_{j}\right\rangle \otimes\left(\sigma_{j}|\psi\rangle\right)$. The Bell measurement 
on the first two qubits collapses the initial state to one of the four terms, and conditioned on the outcome $j, \sigma_{j}$ is applied on the last qubit to recover $|\psi\rangle$.

Teleportation was initially proposed as a communication protocol, but it turns out to be useful in indirect gate constructions. In particular, there are two trivial methods to apply a gate $U$ to a state $|\psi\rangle$. One can teleport $|\psi\rangle$ and then apply $U$ :

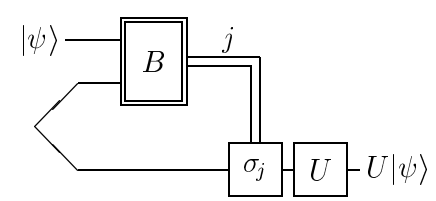

which implies the validity of the following circuit:

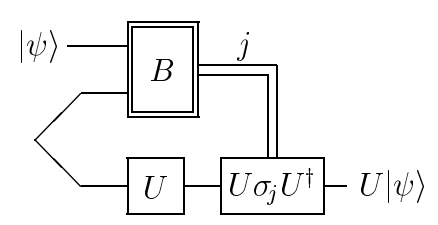

Throughout the paper, $\dagger, T$, and $*$ denote the adjoint, the transpose, and the complex conjugate of an operator respectively. Note that the gate $U \sigma_{j} U^{\dagger}$ is the same as the gates $U^{\dagger}, \sigma_{j}$, and $U$ applied in order. Equation (5) is a recipe for an indirect implementation of $U$ by preparing an "ancilla" $\frac{1}{\sqrt{2}}(I \otimes U)(|00\rangle+|11\rangle)$ and applying a Bell measurement and a "correction" $U \sigma_{j} U^{\dagger}$. It is indirect in that, one must provide the ancilla and the correction with resources allowed in the context, without applying $U$ (see Refs. 9, 5, 10, 11, 13] 17] in the contexts of fault-tolerance and alternative computation models).

The second trivial method to apply $U$ to $|\psi\rangle$ is to apply $U$ and teleport $U|\psi\rangle$ :[24]

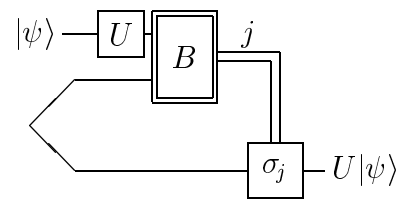

Applying $U$ on the first register followed by the Bell measurement is equivalent to applying a measurement $B_{U_{1}^{\dagger}}$ along the basis $\left\{\left(U^{\dagger} \otimes I\right)\left|\Phi_{j}\right\rangle\right\}_{j}$ :

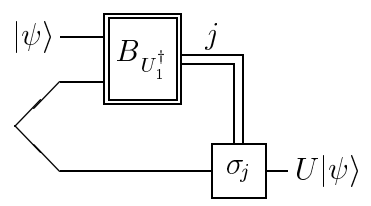


Equations (5) and (7) also generalize trivially to any $n$-qubit gate. We focus on the 2-qubit generalizations:
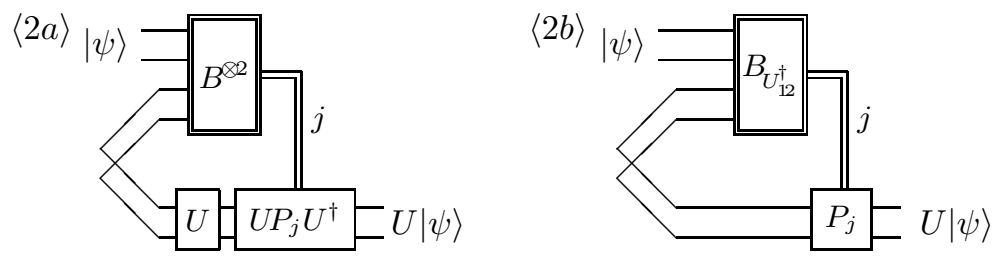

In Eq. (8) $\langle 2 a\rangle, B^{\otimes 2}$ stands for $B_{13} \otimes B_{24}$, a Bell measurement on qubits 1,3 and one on qubits 2, 4. In Eq. (8) $\langle 2 b\rangle$, we have combined $U$ and $B^{\otimes 2}$ in a single measurement $B_{U_{12}^{\dagger}}$ along the basis $\left\{\left(U_{12}^{\dagger} \otimes I_{34}\right)\left(\left|\Phi_{j_{1}}\right\rangle_{13} \otimes\right.\right.$ $\left.\left.\left|\Phi_{j_{2}}\right\rangle_{24}\right)\right\}_{j_{1}, j_{2}}$. We denote a 2-qubit Pauli operator as $P_{j}=\sigma_{j_{1}} \otimes \sigma_{j_{2}}$ where $j \equiv\left(j_{1}, j_{2}\right)$. The following variants of Eqs. (5) and (7) will also be useful:
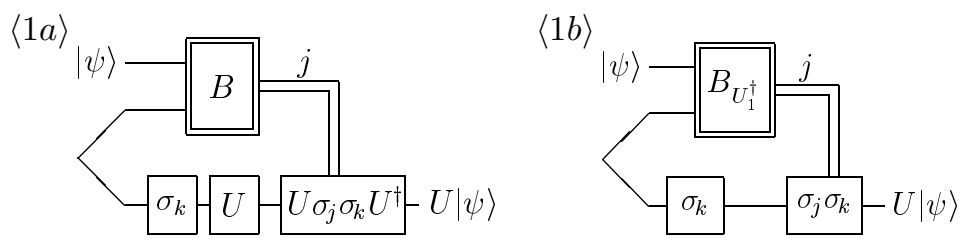

\section{Quantum Computation by measurements only}

Given the ability to perform projective measurements, state initialization and final readout are trivial, and according to the standard model, it remains to perform a universal set of gates. We will explain in detail how to use measurements to provide the various resources needed in the schemes $\langle 1 a, b\rangle$ and $\langle 2 a, b\rangle$ in Eqs. (8) and (9) to perform nontrivial unitary operations that form a universal set. The identity and swAP operations are implicitly provided by quantum storage and by the ability to choose which qubit to measure.

We first demonstrate how to perform a Pauli operation $\sigma_{l}$ using Bell measurements only. This illustrates some of the basic ideas, and the Pauli operations will also be used repeatedly in subsequent discussions. Consider method $\langle 1 b\rangle$ in Eq. (9). The ancilla can be taken to be $\left(I \otimes \sigma_{k}\right)\left|\Phi_{0}\right\rangle=\left|\Phi_{k}\right\rangle$ for any $k$, and can be obtained as a post-measurement state of a single Bell measurement on any 2-qubit system. When $U=\sigma_{j}$, $B_{U_{1}^{\dagger}}$ along the basis $\left\{\left(U^{\dagger} \otimes I\right)\left|\Phi_{j}\right\rangle\right\}$ is just the Bell measurement (outcomes redefined). With probability $1 / 4$, $j=k$ and no correction gate is needed, in which case the desired $\sigma_{l}$ is performed with 2 Bell measurements. Otherwise, the Pauli correction can be performed recursively, again completed with probability $1 / 4$ when no further correction is needed. One repeats the recursion until correction is unnecessary, which on average occurs after 4 trials and 8 Bell measurements. This performs a deterministic Pauli operation with variable resources.

\subsection{Universality of 4-qubit measurements}

We now give a simple derivation that 4-qubit measurements are universal[17] by using them to perform any 2-qubit gate $U$ via method $\langle 2 b\rangle$ in Eq. (8). We use Bell measurements to perform the Pauli correction and to provide the ancilla, which can be any two Bell states by redefining the correction operation similar to method $\langle 1 b\rangle$ in Eq. (9). Finally, $B_{U_{12}^{\dagger}}$ is a 4 -qubit measurement directly available. 


\subsection{Universality of 2-qubit measurements}

We now demonstrate the universality of 2-qubit measurements. [20] To do this, we consider the simpler universal set of all 1-qubit gates and the CNOT. The 1-qubit gates can be performed using method $\langle 1 b\rangle$ in Eq. (9), which requires 2-qubit measurements only. We use method $\langle 2 a\rangle$ to perform CNOT to avoid applying the unavailable 4-qubit measurement $B_{U_{12}^{\dagger}}$ to the input state. This comes at the cost of two extra complications. First, the correction gate becomes CNOT $\left(\sigma_{j_{1}} \otimes \sigma_{j_{2}}\right)$ CNOT, but this is just a tensor product of Pauli operators since CNOT is in the Clifford group. The second complication and the last obstacle is the need to obtain the special ancilla,

$$
\begin{aligned}
\left|a_{\mathrm{cn}}\right\rangle & =\frac{1}{2}(I \otimes I \otimes \mathrm{CNOT})(|0000\rangle+|0101\rangle+|1010\rangle+|1111\rangle) \\
& =\frac{1}{2}(|0000\rangle+|0101\rangle+|1011\rangle+|1110\rangle) .
\end{aligned}
$$

We prove the universality of 2-qubit projective measurements by showing how they can be used to prepare $\left|a_{\mathrm{cn}}\right\rangle$. For simplicity, we focus on measurement outcomes that correspond to the postmeasurement state $\left|a_{\mathrm{cn}}\right\rangle$. We will see later other outcomes correspond to equally good ancillas. We first present the method in the state representation:

1. Create $\frac{1}{2}(|0\rangle+|1\rangle) \otimes|0\rangle \otimes(|00\rangle+|11\rangle)$ with 1- and 2-qubit measurements.

2. Apply to qubits 2,3 the measurement with 2 projectors:

$$
\begin{aligned}
& P_{+}=\left|\Phi_{0}\right\rangle\left\langle\Phi_{0}|+| \Phi_{1}\right\rangle\left\langle\Phi _ { 1 } \left|=\frac{1}{2}(|00\rangle+|11\rangle)\left(\left\langle00|+\langle 11|)+\frac{1}{2}(|01\rangle+|10\rangle)(\langle 01|+\langle 10|),\right.\right.\right.\right. \\
& P_{-}=\left|\Phi_{2}\right\rangle\left\langle\Phi_{2}|+| \Phi_{3}\right\rangle\left\langle\Phi _ { 3 } \left|=\frac{1}{2}(|00\rangle-|11\rangle)\left(\left\langle00|-\langle 11|)+\frac{1}{2}(|01\rangle-|10\rangle)(\langle 01|-\langle 10|) .\right.\right.\right.\right.
\end{aligned}
$$

When the outcome corresponds to $P_{+}$, the state becomes $\frac{1}{2 \sqrt{2}}(|0\rangle+|1\rangle) \otimes(|000\rangle+|011\rangle+|101\rangle+|110\rangle)$.

3. Measure the parity of qubits 1,3 . If the outcome is even, the state becomes $\frac{1}{2}(|0000\rangle+|1011\rangle+|0101\rangle+$ $|1110\rangle)$, which is $\left|a_{\mathrm{cn}}\right\rangle$.

We can also explain the above scheme in the stabilizer language. 21, 25] The stabilizer of an $n$-qubit state $|\psi\rangle$ is an abelian group with $n$ generators $O_{i}$ such that $O_{i}|\psi\rangle=|\psi\rangle$. These generators specify the state up to a phase. If $O|\psi\rangle=|\psi\rangle, U O U^{\dagger}(U|\psi\rangle)=U|\psi\rangle$, therefore, the state evolves as $|\psi\rangle \rightarrow U|\psi\rangle$ when each generator evolves as $O \rightarrow U O U^{\dagger}$. Furthermore, suppose $M$ is a traceless operator with eigenvalues \pm 1 , and it commutes or anticommutes with each generator. If the outcomes \pm 1 are obtained when measuring $M$, the generators that anticommute with $M$ evolve as $\left\{N_{1}, N_{2}, N_{3}, \cdots\right\} \rightarrow\left\{ \pm M, N_{1} N_{2}, N_{1} N_{3}, \cdots\right\}$.

The stabilizer of $\left|\Phi_{0}\right\rangle_{1,3} \otimes\left|\Phi_{0}\right\rangle_{2,4}$ is generated by $X I X I, Z I Z I, I X I X, I Z I Z$, where $X I X I=\sigma_{x} \otimes I \otimes \sigma_{x} \otimes I$ and so on. Since

$$
\begin{array}{ll}
\text { CNOT }(X I) \text { CNOT }=X X, & \text { CNOT }(I X) \text { CNOT }=I X, \\
\text { CNOT }(Z I) \text { CNOT }=Z I, & \text { CNOT }(I Z) \mathrm{CNOT}=Z Z,
\end{array}
$$

the stabilizer of $\left|a_{\mathrm{cn}}\right\rangle$ is generated by:

$X I X X, Z I Z I, I X I X, I Z Z Z$. 
One can prepare a state by measuring the generators of its stabilizer. However, any generator set for $\left|a_{\mathrm{cn}}\right\rangle$ contains elements of weight 3 (the weight is the number of nontrivial tensor components). Our strategy is to start with an initial state with generators of weights 1 and 2 (step 1) and apply 2-qubit measurements $I X X I$ and then $Z I Z I$ to induce multiplications between generators that anticommute with the measured operator, thereby increasing the weights of the generators. Assuming +1 outcomes, the evolution is given by:

$\begin{array}{cccccccc}X I & I I & \text { measure } & X I & I I & \text { measure } & Z I & Z I \\ I Z & I I & I X X I & I X & X I & Z I Z I & X X & X I \\ I I & X X & \longrightarrow & I I & X X & \longrightarrow & X I & X X \\ I I & Z Z & & I Z & Z Z & & I Z & Z Z\end{array}$.

The final set of generators is equivalent to that in Eq. (13) because multiplying one generator to another does not affect the stabilizer.

We have focused on measurement outcomes that result in $\left|a_{\mathrm{cn}}\right\rangle$ in the above discussion. Other outcomes result in states of the form $\left(\sigma_{k} \otimes \sigma_{l} \otimes \mathrm{CNOT}\right)\left|\Phi_{0}\right\rangle_{1,3} \otimes\left|\Phi_{0}\right\rangle_{2,4}= \pm\left(I \otimes I \otimes\left(\mathrm{CNOT} \sigma_{k} \otimes \sigma_{l}\right)\right)\left|\Phi_{0}\right\rangle_{1,3} \otimes\left|\Phi_{0}\right\rangle_{2,4}$ (which can be used as the ancilla by adapting the correction procedure as in Eq. (9)). This is immediate in the stabilizer representation - post-measurement states of other outcomes differ by extra - signs in some of the generators. These signs can be induced by applying Pauli operators to the first 2 qubits of $\left|a_{\mathrm{cn}}\right\rangle$. Thus other output states are precisely $\left(\sigma_{k} \otimes \sigma_{l} \otimes I I\right)\left|a_{\mathrm{cn}}\right\rangle$. This can also be verified directly in the state representation.

We turn our attention to discrete universal sets of (incomplete) 2-qubit measurements that correspond to discrete universal sets of gates. It is known that the Clifford group generated by $\{\mathrm{CNOT}, \mathrm{H}, \mathrm{P}\}$ together with any other gate are universal [26]. Thus $\{$ CNOT, H, P, U $\}$ is universal for any 1-qubit gate U outside the Clifford group. We can enumerate all the required measurements. First, all correction gates are Pauli operators, requiring only Bell measurements (i.e. measuring $X X$ and $Z Z$ ). We need to perform $B_{\mathrm{H}_{1}^{\dagger}}, B_{\mathrm{U}_{1}^{\dagger}}$, and $B_{\mathrm{P}_{1}^{\dagger}}$ for the 1-qubit gates. In general, $B_{U_{1}^{\dagger}}$ is a measurement of the operators $\left(U^{\dagger} X U\right) \otimes X$ and $\left(U^{\dagger} Z U\right) \otimes Z$. Thus we need to measure $X Z, X Y$, and $\left(\mathrm{U}^{\dagger} X \mathrm{U}\right) \otimes X$ and $\left(\mathrm{U}^{\dagger} Z \mathrm{U}\right) \otimes Z$ to perform H, $\mathrm{P}$, and $\mathrm{U}$ respectively. Finally, ancilla preparation requires other measurements. The Bell state ancilla for any 1-qubit gate requires no new measurement. The preparation of the state $\left|a_{\mathrm{cn}}\right\rangle$ for CNOT requires the states $|0\rangle$ and $\frac{1}{\sqrt{2}}(|0\rangle+|1\rangle)$. We can measure $Z$ to prepare $|0\rangle$, and apply H to $|0\rangle$ to obtain $\frac{1}{\sqrt{2}}(|0\rangle+|1\rangle)$. Altogether,

$$
S_{0}=\left\{Z, X X, Z Z, X Z, X Y,\left(\mathrm{U}^{\dagger} X \mathrm{U}\right) \otimes X,\left(\mathrm{U}^{\dagger} Z \mathrm{U}\right) \otimes Z\right\}
$$

is universal. Special choices of u can further simplify the universal set. For instance,

$$
\begin{aligned}
& S_{1}=\{Z, X X, Z Z, X Z, X Y,(\cos \theta Z+\sin \theta Y) \otimes Z\} \\
& S_{2}=\{Z, X X, Z Z, X Z, X Y,(\cos \theta X+\sin \theta Y) \otimes X\} \\
& S_{3}=\left\{Z, X X, Z Z, X Z, \frac{1}{\sqrt{2}}(X+Y) \otimes X\right\}
\end{aligned}
$$

are universal sets of measurements corresponding to $\mathrm{U}=e^{i \frac{\theta}{2} X}, e^{-i \frac{\theta}{2} Z}$, and $e^{-i \frac{\pi}{8} Z}$ respectively $(\theta \neq m \pi / 2$ for $m$ an integer). $S_{3}$ corresponds to the universal set of gates $\left\{e^{-i \frac{\pi}{8} Z}, \mathrm{H}, \mathrm{CNOT}\right\}$,

The above scheme implements a desired gate precisely at each stage of a computation by performing the Pauli 
correction with variable resources. However, this is unnecessary. Suppose the sequence of gates $\left\{V_{1}, V_{2}, \cdots\right\}$ are to be applied to the $i$-th qubit, where $V_{j}$ is a 1-qubit gate or a CNOT (involving another qubit). Instead of applying the correction $\sigma_{i_{1}}$ for $V_{1}$, we can absorb $\sigma_{i_{1}}$ into the next gate $V_{2}$, i.e., to perform $V_{2} \sigma_{i_{1}}$ instead. If $V_{2}$ is in the Clifford group, then, $V_{2} \sigma_{i_{1}}=\sigma_{i_{1}^{\prime}} V_{2}$ for some $i_{1}^{\prime}$ and we simply perform $V_{2}$. If the correction for $V_{2}$ is $\sigma_{i_{2}}$, the combined correction is $\sigma_{i_{1}^{\prime}} \sigma_{i_{2}}$. Thus the correction for $V_{1}$ can be omitted by redefining the correction for $V_{2}$. The combined $\sigma_{i_{1}^{\prime}} \sigma_{i_{2}}$ can now be absorbed in $V_{3}$ similarly. If $V_{2}$ is not in the Clifford group, then $V_{2}=\mathrm{U}$ is a 1-qubit gate, and we perform $\mathrm{U} \sigma_{i_{1}}$ by replacing $B_{\mathrm{U}_{1}^{\dagger}}$ in method $\langle 1 b\rangle$ (Eq. (9)) by $B_{\left(\sigma_{i_{1}} \mathrm{U}\right)_{1}^{\dagger}}$. Now, our universal sets of measurements $S_{1,2,3}$ each require an extra element similar to the last element listed for each set.

\subsection{Universality of a single 4-qubit measurement}

We turn to a different task that parallels the search of a single 2-qubit gate that is universal. [8, 6, 17. We will show that the 4-qubit measurement $B_{U_{12}^{\dagger}}$ alone is universal for appropriately chosen $U$. We denote the Pauli group over 2 qubits by $\mathcal{P}_{2}$, with elements $P_{j}=\sigma_{j_{1}} \otimes \sigma_{j_{2}}, j \equiv\left(j_{1}, j_{2}\right)$. We first find out what gates can be performed by using the measurement $B_{U_{12}^{\dagger}}$. According to Eq. (8), the following circuits are valid:

(A)

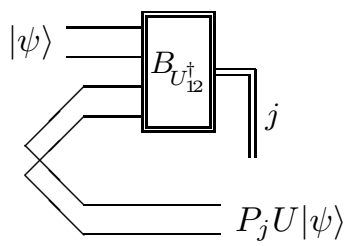

(B)

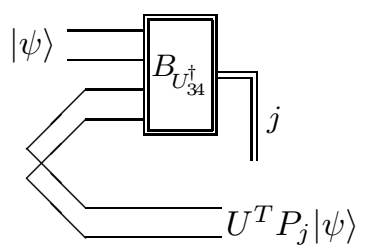

Circuit (A) is derived from method $\langle 2 b\rangle$ in Eq. (8) without correcting for $P_{j}$. In circuit (B) $B_{U_{34}^{\dagger}}$ denotes the same measurement as $B_{U_{12}^{\dagger}}$ with qubits $(1,2)$ and $(3,4)$ interchanged. This measurement has the same effect as $U$ acting on qubits 3,4 followed by Bell measurements $B_{13} \otimes B_{24}$. The circuit follows from teleportation and the fact that $U$ acting on half of a maximally entangled state is the same as $U^{T}$ acting on the other half.

Bell states used in Eq. (14) are not directly available in the current task. Instead, we use the following ancillas obtainable from $B_{U_{12}^{\dagger}}$ :

(C)

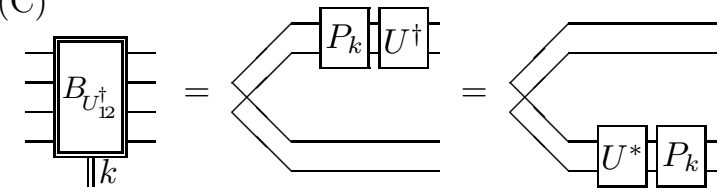

(D)

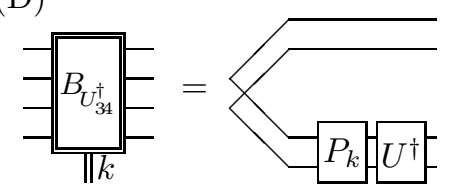

In particular, we can perform some gates using the following two primitives :

1. If ancilla (D) is used in place of the Bell states in circuit (A), the output state will be $U^{\dagger} P_{k} P_{j} U|\psi\rangle$. Thus one can apply $U^{\dagger} P_{k} P_{j} U$ to any 2-qubit state.

2. Likewise, replacing the Bell states in circuit (B) by ancilla (C), the final state is $P_{k} U^{*} U^{T} P_{j}|\psi\rangle=$ $P_{k} P_{j}|\psi\rangle$, thus $P_{k} P_{j}$ is applied to the input state. 
Using primitive (2), a random element from the set $\left\{P_{k}\right\}_{k}$ can be performed. The randomness can be removed. Repeating primitive (2) $l$ times results in the gate $G_{l}=P_{k_{l}} \cdots P_{k_{2}} P_{k_{1}} \in \mathcal{P}_{2} .\left\{G_{l}\right\}$ is a random walk on $\mathcal{P}_{2}$ that hits any desired $P \in \mathcal{P}_{2}$ with an average of 16 iterations. Likewise, repeated use of primitive (1) results in a similar random walk $U^{\dagger} P_{k_{l}} \cdots P_{k_{2}} P_{k_{1}} U$ allowing $U^{\dagger} P U$ to be performed for any desired $P$.

In particular, $Q U^{\dagger} P U$ can be performed for any $Q, P \in \mathcal{P}$ of our choice. We claim that $Q U^{\dagger} P U$ is universal when $P=Q=I \otimes Z$,

$$
U=\left[\begin{array}{l|l}
I_{2} & 0_{2} \\
\hline 0_{2} & R
\end{array}\right]
$$

where $I_{2}, 0_{2}$ are the $2 \times 2$ identity and zero matrices respectively, and

$$
R=\left[\begin{array}{lr}
\cos \theta & -i e^{-i \phi} \sin \theta \\
-i e^{i \phi} \sin \theta & \cos \theta
\end{array}\right]
$$

is a rotation of $2 \theta$ about the axis $\cos \phi X+\sin \phi Y$. Then,

$$
Q U^{\dagger} P U=\left[\begin{array}{c|c}
Z & 0_{2} \\
\hline 0_{2} & Z
\end{array}\right] \times\left[\begin{array}{c|c}
I_{2} & 0_{2} \\
\hline 0_{2} & R^{\dagger}
\end{array}\right] \times\left[\begin{array}{c|c}
Z & 0_{2} \\
\hline 0_{2} & Z
\end{array}\right] \times\left[\begin{array}{c|c}
I_{2} & 0_{2} \\
\hline 0_{2} & R
\end{array}\right]=\left[\begin{array}{c|c}
I_{2} & 0_{2} \\
\hline 0_{2} & \left(Z R^{\dagger} Z\right) R
\end{array}\right] .
$$

Note that $Z R^{\dagger} Z=R$ because $Z$ anticommutes with both $X$ and $Y$ and reverses $R^{\dagger}$. Thus, $Q U^{\dagger} P U=U^{2}$, which is universal if $\theta$ and $\phi$ are both irrational multiples of $\pi$, and their ratio is also irrational. [8]

The measurement $B_{U_{1}^{\dagger}}$ is actually universal for almost all 2-qubit gates $U$. This is because the spectra of $H$ where $e^{-i H}=Q U^{\dagger} P U$ form a set of positive measure in $\mathbb{R}^{4}$ when $P, Q, U$ are varied, while the set of all nonuniversal 2-qubit gates is of zero measure. [6. 17. On the other hand, denote the Clifford group by $C_{2}$ and the set that conjugates the Pauli group into the Clifford group by $C_{3}$. [10] If $U=U_{1} U_{2}^{\dagger}$ for $U_{1}, U_{2} \in C_{3}$. Our scheme only generates $P_{1} U^{\dagger} P_{2} U P_{3} \cdots$ which is always in $P U_{2} C_{2} U_{2}^{\dagger} Q$.

\section{Conclusions}

We described a variety of methods for performing gates by measurements only. We showed that 2-qubit measurements are necessary and sufficient for universal quantum computation. This is optimal in the number of qubits to be measured jointly. We proved the universality of almost all single maximally entangling 4qubit measurements. This is minimal in the number of measurements available. Method $\langle 1 b\rangle$ (and its $n$-qubit generalization) differs from previous methods $(\langle 1 a\rangle)$ in that it only requires Pauli correction. This allows the removal of the correction procedure in some of our schemes as in the 1-way quantum computer, [15] providing further hint that the two measurement models are related.

Various open questions remain. We will explore the relation between the two measurement models in the future. Universality of measurements that are not maximally entangling remains to be investigated. Finally, error correction, fault-tolerance, and error thresholds remain to be investigated in detail.

Though experimental advantages of measurement-based quantum computation are yet to be found, alternative models for quantum computation and their universality requirements are important for new experimental directions and insights on what makes quantum computation powerful. 


\section{Acknowledgments}

We thank Michael Nielsen and David DiVincenzo for interesting discussions motivating part of the current result. Jim Harrington corrected a mistaken omission of $Z$ in $S_{0,1,2,3}$, and Daniel Gottesman drew our attention to Ref. [26]. We thank Allen Knutson and Eric Rains for insightful ideas on the universality of the set $\left\langle P, U^{\dagger} P U\right\rangle_{P \in \mathcal{P}_{2}}$. DWL is indebted to Charles Bennett, Isaac Chuang, Beth Ruskai, John Smolin, and Barbara Terhal for helpful discussions and encouragements. Duncan Mortimer made an extensive study of measurement models in his senior thesis, University of Queensland, 2002. Part of this work was completed when DWL was at IBM TJ Watson Research Center and at ITP, UCSB. DWL is partially supported by the US NSF under grant no. EIA-0086038 and by the Richard C. Tolman Endowment Fund at Caltech.

\section{References}

[1] D. P. DiVincenzo, Science 270, 255 (1995).

[2] See chapter 4 in 22] for a summary of the major results and historical notes. A partial list of original contributions include Refs. 3, 4, 5, 6, 7, 8,

[3] D. P. DiVincenzo, Phys. Rev. A 51, 1015 (1995).

[4] A. Barenco, C. Bennett, R. Cleve, D. DiVincenzo, N. Margolus, P. Shor, T. Sleator, J. Smolin, and H. Weinfurter, Phys. Rev. A 52, 3457 (1995).

[5] P. O. Boykin et al., in Proc. $40^{\text {th }}$ Annual Symposium on Foundations of Computer Science (IEEE Computer Society Press, Los Alamitos, CA, 1999).

[6] S. Lloyd, Phys. Rev. Lett. 75, 346 (1995).

[7] D. Deutsch, A. Barenco, and A. Ekert, Proc. R. Soc. London A 449, 669 (1995).

[8] A. Barenco, quant-ph/9505016

[9] P. Shor, in Proc. 37th Annual Symposium on Foundations of Computer Science (IEEE Computer Society Press, Los Alamitos, CA, 1996), p. 56.

[10] D. Gottesman and I. Chuang, Nature 402, 390 (1999).

[11] X. Zhou, D. Leung, and I. Chuang, Phys. Rev. A' 62, 052316 (2000).

[12] C. H. Bennett, G. Brassard, C. Crépeau, R. Jozsa, A. Peres, and W. K. Wootters, Phys. Rev. Lett. 70, 1895 (1993).

[13] E. Knill, R. Laflamme, and G. Milburn, Nature 409, 26 (2001).

[14] L.-A. Wu and D. Lidar, Phys. Rev. A 67, 050303 (2003).

[15] R. Raussendorf and H. J. Briegel, Phys. Rev. Lett. 86, 5188 (2001).

[16] H. J. Briegel and R. Raussendorf, Phys. Rev. Lett. 86, 910 (2001).

[17] M. A. Nielsen, quant-ph/0108020v1. 
[18] S. A. Fenner and Y. Zhang, quant-ph/0111077,

[19] Leung proved that 3-qubit measurements are universal (guest lecture, MIT MAS.961, Oct. 2001) because CNOT only has Pauli corrections and its ancilla can be prepared by measuring $I Z Z Z$ on $\frac{1}{2 \sqrt{2}}(|00\rangle+$ $|11\rangle)(|0\rangle+|1\rangle)^{\otimes 2}$. Nielsen subsequently noted that the ancilla for any 2-qubit gate has stabilizer generators of weight $\leq 3$, and can be prepared by 3 -qubit measurements.

[20] D. Leung, quant-ph/0111122

[21] D. Gottesman, Ph.D. thesis, CalTech, Pasadena, CA, 1997, quant-ph/9705052

[22] M. A. Nielsen and I. L. Chuang, Quantum computation and quantum information (Cambridge University Press, Cambridge, U.K., 2000).

[23] D. W. Leung, Ph.D. thesis, Stanford University, Palo Alto, CA, 2000, cs.CC/0012017

[24] This trick has been implicitly used by Gottesman [21] to construct fault tolerant Clifford group gates for general stabilizer codes.

[25] D. Gottesman, quant-ph/9807006.

[26] G. Nebe, E. Rains, and N. Sloane, math.CO/0001038. 